Satoko Abe · Kumiko Koyama • Shin-ichi Usami

Yusuke Nakamura

\title{
Construction and characterization of a vestibular-specific cDNA library using T7-based RNA amplification
}

\begin{abstract}
Using a very small amount of inner-ear tissue, we constructed a human vestibular cDNA library by means of T7-based amplification of RNA. This library should allow us to identify genes likely to be involved in auditory and vestibular functions. Here we first report the characterization of the human vestibular cDNA library. Among 506 cDNA clones randomly selected from the vestibular cDNA library, DNA sequences of $301 \mathrm{cDNA}$ clones were identical to those of genes of known function. Twenty-two cDNA clones were considered to be novel because they did not match any cDNA sequences in the public database. The information in our study will provide a valuable resource for identifying several novel genes underlying deafness disorders and vestibular dysfunction.
\end{abstract}

Key words Human vestibule $\cdot$ cDNA library $\cdot$ T7-based RNA amplification - Hearing loss $\cdot$ Vestibular dysfunction

\section{Introduction}

Analysis of the human genome at the molecular level has made it possible to identify genes responsible for hereditary deafness; to date investigators have cloned 28 genes associated with nonsyndromic deafness and dozens involved in deafness syndromes (multiple phenotypic disease).

Among several different approaches for identifying aberrant genes responsible for hearing disorders, the most common strategy has been the positional-candidate ap-

S. Abe $\cdot$ K. Koyama $\cdot$ Y. Nakamura $(\bowtie)$

Laboratory of Molecular Medicine, Human Genome Center,

Institute of Medical Science, The University of Tokyo, 4-6-1

Shirokanedai, Minato-ku, Tokyo 108-8639, Japan

Tel. +81-3-5449-5372; Fax +81-3-5449-5433

e-mail: yusuke@ims.u-tokyo.ac.jp

S. Usami

Department of Otorhinolaryngology, Shinshu University School of

Medicine, Matsumoto, Japan proach coupled with gene-mapping information and genetic linkage analysis, using selected groups of affected and unaffected individuals. Many genes, including GJB2, TECTA, and $O T O F$, have been identified in this way (Kelsell et al. 1997; Verhoeven et al. 1999; Yasunaga et al. 1999). Although this method is very effective for discovery of novel deafness-related genes, narrowing a candidate locus to a very small interval requires participation of relatively large families and comprehensive clinicopathological data. Another approach is based on specific expression patterns or functions in target organs. Inner-ear cDNA libraries have been constructed from multiple samples of fetal cochlear tissue to isolate genes associated with hearing (Robertson et al. 1994; Jacob et al. 1997). This approach has identified three genes responsible for deafness: $A T Q 1$, COCH, and OTOR (Robertson et al. 1994, 1997, 2000; Skvorak et al. 1997). A large series of cochlea organ analysis has been performed, including more than 4000 human expressed sequence tags (ESTs) from the fetal cochlear library deposited in the public database (see http:// hearing.bwh.harvard.edu/cochlearcdnalibrary.htm). However, to date, no attempt has been made to characterize the expressed genes of the human vestibule representing a functionally essential compartment in the inner ear. Vestibular symptoms are clinically either sole complaints or sometimes associated with deafness. Among the cochleo-vestibular dysfunctions, Meniere's disease, which is defined as a clinically representative inner ear disorder accompanied by sensorineural hearing loss and vertigo, was dominantly inherited in some families (Fung et al. 2002). The importance of genetic factors in Meniere's disease has been suggested, but very few genes associated with vestibular dysfunction have been cloned. The $\mathrm{COCH}$ gene, which is predominantly expressed in the inner ear, is demonstrated to be responsible for one type of nonsyndromic hearing loss with progressive hearing loss and vestibular dysfunction (Robertson et al. 1998). In contrast to the recent progress in the discovery of genes responsible for hearing loss, genes related to vestibular symptoms are not yet fully understood. Although many similarities are observed in the general features of cochlea and vestibular end organs, there are consid- 
erable differences in these homologous organs, including their evolution, structure, and function.

Therefore, as an additional resource for investigating genes essential for hearing and equilibrium, we constructed a human vestibular cDNA library by means of T7-based amplification of RNA from a very small amount of adult inner-ear tissue (Luo et al. 1999), and here describe the detailed analytical results obtained from reverse transcriptase-polymerase chain reaction (RT-PCR) experiments. To our knowledge, this is the first study of the gene expression profiles of human vestibular tissue.

\section{Materials and methods}

\section{RNA extraction and T7-based amplification}

Tissue samples of an adult vestibule were obtained from a single patient during labyrinthectomy with prior written informed consent, and immediately stored at $-80^{\circ} \mathrm{C}$. Total RNA was extracted from each tissue with an RNeasy mini kit (Qiagen, Valencia, CA, USA). Because of the extremely small amount of vestibular total RNA available at nanogram-level quantities, we applied one cycle of T7based RNA amplification after DNase I treatment of total RNA and subsequent cDNA synthesis, as described previously (Luo et al. 1999), with some modifications.

\section{Construction and analysis of a vestibular cDNA library}

For second-strand cDNA synthesis, a linker-primer that included an internal XhoI site (5'-AGTCTCGAGTCTAG TCGACGGCCAGTGAATTGTAATACGACTCACTATAGG $\left.\mathrm{GCGT}_{21}-3^{\prime}\right)$ was used. After double-strand cDNA fragments of less than $200 \mathrm{bp}$ were removed by the use of CHROMA spin column-400 (CLONTECH, Palo Alto, CA, USA), a cDNA library was constructed using the cDNA Synthesis kit (Stratagene, LaJolla, CA, USA) according to the supplier's instructions. From recombinant clones, we randomly selected 506 clones and determined their DNA sequences. The nucleotide sequence of each clone was compared with those of cDNA sequences in the GenBank database of the National Center for Biotechnology Information as of October 2002. The clones were classified according to the entries in GenBank (Table 1).

Table 1. Classification of cDNA sequences from the vestibular tissue

\begin{tabular}{lc}
\hline Database match & $n(\%)$ \\
\hline Number of clones sequenced & 506 \\
Known function genes & $301(59.5)$ \\
Repetitive sequences & $139(27.5)$ \\
Clones identical to reported ESTs & $29(5.7)$ \\
Novel ESTs & $22(4.3)$ \\
Mitochodrial DNA sequences & $15(3.0)$
\end{tabular}

EST, Expressed sequence tag

\section{RT-PCR analysis}

From among the 506 EST sequences obtained from the vestibular cDNA library, we selected clones that showed no match to any cDNA sequences in the database. Twenty-two novel EST sequences were submitted to the Center for Information Biology and DNA Data Bank of Japan (DDBJ); their GenBank accession numbers are AB0728187, AB072818, AB077323, AB074042, AB076975, AB074038, AB074039, AB072821-AB072825, AB074046, AB07673, AB074133, AB074135, AB076955, AB076958, AB076959, AB076961, AB076962, and AB076966. To determine the tissue expression patterns of the selected ESTs, we examined them by semiquantitative RT-PCR analysis using one round of T7-based amplified cDNAs from one vestibular organ and eight organs (brain, heart, placenta, lung, liver, skeletal muscle, kidney, and pancreas). Human glceraldehyde-3-phosphate dehydrogenase $(G A P D H)$ was used as a quantity control. Sets of primers corresponding to each gene were designed from nucleotide sequences of the vestibular cDNA clones obtained, and these sets were first tested on genomic DNA to test the PCR. Amplification was performed at an annealing temperature of $95^{\circ} \mathrm{C}$ for $2 \mathrm{~min}$, followed by $30-35$ cycles of $95^{\circ} \mathrm{C}$ for $30 \mathrm{~s}, 55^{\circ} \mathrm{C}$ for $30 \mathrm{~s}$, and $72^{\circ} \mathrm{C}$ for $30 \mathrm{~s}$. The annealing temperature was adjusted according to the Tm of the primers used. PCR products were electrophoresed on a $2 \%-3 \%$ agarose/Ethidium bromide gel.

\section{Results}

Evaluation and functional categorization of the vestibular cDNA library

The cDNA library was constructed from membranous labyrinths (vestibule) from adult inner ear. Very careful microscopic dissection was performed to avoid contamination by surrounding bony, cartilaginous, and fibrous elements. We obtained approximately $2.7 \times 10^{4}$ plaques and performed a partial characterization using 506 randomly selected clones. The insert sizes of these cDNA clones ranged from 0.2 to $0.5 \mathrm{kbp}$. Their DNA sequences were compared with sequences in the public database and classified as shown in Table 1. Of the 506 clones sequenced, 301 (222 independent clones), representing $59.5 \%$ of the total, were identical to sequences of genes whose functions were known; 29 (5.7\%) were identical to archived EST sequences; 15 (3.0\%) were identical to mitochondrial sequences; and 22 (4.3\%) did not match any cDNA sequences in the public database. DNA sequences of all or part of the remaining 139 clones (27.5\%) were repetitive elements. Genes corresponding to ESTs are summarized in Table 2. Comparison of our 222 independent vestibular genes of known functions with DNA sequences from a fetal cochlear library reported previously (Skvorak et al. 1999) revealed that 53 of these cDNAs were common to both studies (Table 2). The vestibular cDNA sequences that were identical to genes of known function were classified into 17 categories according to their functional 
Table 2. The functional categories of known genes found in the vestibular cDNA library

Gene description

\begin{tabular}{lll} 
Accession & $\begin{array}{l}\text { Identical to } \\
\text { cochlear } \\
\text { Occurrence }\end{array}$ & ESTs \\
\hline
\end{tabular}

\section{Cell cycle/cell proliferation}

Apoptosis inhibitor survivin

BBP-like protein 1 (BLP1)

bcl-1

Cell cycle regulator protein $\mathrm{p} 95$

Chondroitin sulfate proteoglycan 2 (versican) (CSPG2)

WD repeat domain 7 (WDR7), transcript variant 1 (= TGF-beta)

resistance associated gene)

Programmed cell death-2/RP8 homolog (PDCD2)

HSU75285

AF353991

HUMBCL1

AF058696

NM004385

Cell surface markers/cell adhesion

Integral transmembrane protein

Junctional adhesion molecule 3 (JAM3)

Ligatin

Lysosomal-associated protein transmembrane 4 alpha (LAPTM4A)

Progesterone membrane binding protein (PMBP)

Pro-oncosis receptor inducing membrane injury gene

Putative type 1a integral membrane protein

Retinoic acid receptor responder (tazarotene induced) 1 (RARRES1)

Similar to integral membrane protein 3

Tight junction protein 1

Transmembrane protein 1 (TMEM1)

Transmembrane protein BRI

NM_015285

\section{Extracellular matrix}

Alpha-3 type IV collagen (COL4A3)

Collagen type IV alpha 5 (COL4A5)

Endoplasmic reticulum glycoprotein

\section{Cellular metabolism}

Aminoadipate-semialdehyde dehydrogenase-phosphopantetheinyl transferase

Beta1-4 galactosyl transferase

Carbonic anhydrase II (CA2)

CDP-diacylglycerol synthase (CDS)

Cisplatin resistance associated (CRA)

Cytochrome C oxidase subunit IV precursor (COX4) gene, encoding

mitochondrial protein

Cytosolic selenium-dependent glutathione peroxidase

Diaphorase (NADH) cytochrome b-5 reductase, nuclear gen

encoding $\mathrm{mt}$ protein,

Dioxin inducible cytochrome 450

Ectonucleotide pyrophosphatase/phosphodiesterase 4

Filamin (FLN) gene to glucose-6-phosphate dehydrogenase (G6PD)

FRA3B common fragile region diadenosine triphosphate hydrolase (FHIT)

Glutathione peroxidase 4 (CPX4)

Inducible 6-phosphofructo-2-kinase/fructose 2, 6-bisphos

Lactate dehydrogenase B (LDHB)

p6=cytochrome $\mathrm{c}$ oxidase subunit VIc homolog/COSVIc/prostatic

carcinoma upregulated

Phenylalkylamine binding protein

Prostaglandin D2 synthase gene, exon 7

Putative DNA dependent ATPase and helicase (ATAX)

Pyruvate dehydrogenase E1-alpha subunit

Retinal short-chain dehydrogenase/reductase

Rhodanese (=thiosulfate sulfurtransferase)

Serine palmitoyl transferase (SPTL1)

Sphingomyelin phosphodiesterase

Stannin

Thiopurine methyl transferase (TPMT)

TPI1 gene for thiosephosphate isomerase

Unspliced mRNA for glutathione peroxidase

\section{AL031259}

ITM1

NM_032801

AF159586

NM_014713

NM_006320

HSM 800854

HSS171

RARES1

BC002424

TJP1

XM009794

AF152432

HUMCOL4A3X

U04520

NM_006816

Cytoskeletal proteins

Actin-gamma 1

Alpha-actin

Beta-2-microglobulin

C3 (complement component) (C3) gene $3^{\prime}$-end

Cytokelatin exon, delta-aminolevulinate synthase (erythroid)

Lamin A/C

WAS protein family, member 2

$\begin{array}{ll}\text { AF151838 } & \\ \text { AF038662 } & \\ \text { NM000067 } & + \\ \text { HSU6008 } & \\ \text { AW00589 } & + \\ \text { AF017115 } & + \\ & \\ \text { HUMGLPEX } & + \\ \text { DIA1B } & \\ & \\ \text { HSU56438 } & + \\ \text { NM_014936 } & \\ \text { HUMFLNG6PD } & \\ \text { AF020503 } & \\ \text { GPX4 } & \\ \text { AF056320 } & + \\ \text { LDHB } & \\ \text { S82616 } & +\end{array}$

AF196969

HUMDS03

HSU72938

HUMPDHE1B +

AF126782

D87292

AF286717

X52678

AF286717

HSTHSMT1

HSTPI1G

HSPEROXP

ACTG1
HSAPACT
B2M
M63422
HS884M20
BC003162
AK025566


Table 2. Continued

Gene description

Occurrence

no.

Identical to
cochlear

DNA replication and repair

Topoisomerase II beta

X-ray repair complementing defective repair in Chinese hamster cells 3 (XRCC3)

$\mathrm{U} 65315$

Serum/blood cell proteins

Apoliprotein cluster

B-cells-hypothetical protein

Beta-globin

$\mathrm{hHa} 2$ gene from circulating lymphocyte

Ig gamma heavy chain, membrane-bound type, and secret type

Immunoglobulin heavy chain variable region

Immunogobulin lamda

Neuronal

Ceroid-lipofuscinosis, neural 3, juvenile (CLNS3)

Glioblastoma amplified secreted protein (GASP)

Highly similar to SPECTRIN beta chain, brain

NDRG family member 4 (NDRG4)

Neuroxin III-alpha

Purkinje cell protein 2

Sine oculis homeobox homolog 3 (Drosophila) (SIX3)

XM_050297

HSALINT

AA362439

AF007546

X90761

D78345

HSA7320

D88268

ESTs

Translation/protein synthesis

$28 \mathrm{~S}$ ribosomal RNA

40S ribosomal protein S18

60S ribosomal protein L13a

$60 \mathrm{~S}$ ribosomal protein $\mathrm{L} 15$

60 S ribosomal protein L37A

Heterogeneous nuclear ribosomal protein 11

Ribosomal proteon S6

Ribosomal protein L3

Ribosomal protein L9

Ribosomal protein L15

Ribosomal protein L17

Ribosomal protein L27

Ribosomal protein L35a

Ribosomal protein L37

Ribosomal protein L41

Ribosomal protein L44

Ribosomal protein S11

Ribosomal protein S12

Ribosomal protein S19

Ribosomal protein S24

Ribosomal protein S25

Ribosomal protein S27A (ubiquitin carboxyl extension protein)

Ribosomal protein $\mathrm{S} 7$

Ribosomal protein S23

Surf 3 gene for ribosomal protein L7A

TATA binding protein (TBP)-associated factor

The gene for U2 small nuclear RNA-associated beta antigen

RNA processing

Nucleolar protein family A, member 2 (H/ACA small nucleolar RNPs) (NOLA2)

The alternatively spliced gene for the human orthologs of mouse QKI-7 and QKI-7B

NM000086

AF395824

AK023762

NM_022910

AF099810

S40022

NM_005413

Transcription/nuclear proteins

HIV Tat-SF1

Homeobox protein NKX3.1

Max

Nuclear protein family 3 similar to integral membrane

Nuclear transport factor 2

Transcription factor ETR103

Zinc-finger protein 147 (ZNF 147) (estrogen-responsive finger protein)

AF152922

AL031228

X56932

AF279903

HS495010

HNRPA1

HSRPS6G

HUMRPL3A

RPL9

HS90L6

RPL17

HUMRIBPROE

RPL35A

L11567

AF026844

RPL44

RPS11

NM001016.1

RP19

HSU12202

RPS25

RPS27A

RPS7

RPS23

HSSURF3

TAF2G

HS705D16

NM_017838

HS51J12

U76992

AF247704

HSMAXG

BC002424

BC002348

HUMETR103

XM012593

Kinase and phosphatase

Eph-like receptor tyrosine kinase hEphB1b (EphB1)

PI-3 kinase

PNP1 mRNA (thyrosine phosphatase)

Protein kinase inhibitor gamma (PKIG)

Protein phosphatase 1, gamma

Protein phosphatase 1, catalytic subunit, alpha isoform

AF037332

HSpI3KINK

HSPNP1

AF182032

L07395

BC004482

$+$

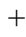

$+$

$+$ 
Table 2. Continued

Gene description

Identical to

Identical to
cochlear

PTPL mRNA for protein tyrosine phosphatase

ESTs

$\mathrm{RYK}=$ related to receptor tyrosine kinase from human hepatoma

HSPTPL1

Similar to serine threonine kinase pim3

YSK1

S59184

BC017083

D63780

Proteolysis

F-box only protein 7

Polyubiquitin UbC

Proteosome (prosome, maropain) subunit, beta type 1

Sorting nexin1A (SNX1)

XM 0099

AB009010

NM 002793

AF065484

Signaling molecules/growth factors/receptors

Amyloid beta (A4) precursor protein (Alzheimer disease)

Calmodulin 1

Cytoplasmic interactor of beta 4 integrin P27BBP protein (ITBG4BP) gene, exon 4, 5, 6, 7

Diaphorase (NADH) (cytochrome b-5 reductase) (DIA1), nuclear gene encoding mt protein

Elongation factor 2

Glutamate receptor, metabotropic 1 (GRM1)

Insulin-like growth factor 2 receptor (IGF2R)

Insulin-like growth factor binding protein 6 (IGFBP6)

Metabotrophic glutamate receptor 6 (mGluR6)

Okadaic acid-inducible and cAMP-regulated phosphoprotein 19

p0071 protein

Solute carrier family 1 (glial high-affinity glutamate transporter), member3 (SLC1A3)

Testosterone-repressed prostate message 2 (TRPM-2) protein gene

Thrombin receptor gene, exon1

Transmembrane receptor protein

Uterin bom besin receptor

Zona pellucida glycoprotein 2 (sperm receptor)

XM009710

JO4046

AF289541

NM007326

HSEF2MR

XM_054004

XM604237

HSA6952

HSU82083

AF084555

HSP0071

SLC1A3

M63379

AF054633

HSTRECP

HSUBR

ZP2

Channel protein

Apamin-sensitive small-conductive calcium-activated potassium channel (hSK2) (KCNN2)

ATPase, $\mathrm{H}^{+}$transporting, lysosomal mRNA

ATPase, $\mathrm{Na}^{+} / \mathrm{K}^{+}$transporting, alpha2 $(+)$polypeptide

Potassium channel, subfamily K, member 6 (TWIK-2)(KCNK6)

Vestibule-1 protein (mechanosensitive ion channel domain containing protein)

Sodium/myo-inositol cotransporter (SLC5A3)

Voltage-dependent anion channel 3 (VDAC3)

Voltage-dependent calcium channel beta2 subunit (CACNB2)

AF239613

ATP6C

ATP1A2

KCNK6

$\mathrm{AB} 056652$

AF027153

$\mathrm{BC} 002456$

AF465485

\section{Miscellaneous}

40-kDa keratin intermediate filament precursor

6.2-kDa protein

Arg/Abl-interacting protein ArgBP2 (ARGBP2), transcript variant 1

Arg/Abl-interacting protein ArgBP2 (ARGBP2), transcript variant 2

ATPase, class VI, type 11B (ATP11B)

CGI-56 protein

Chemokine-like factor super family 8 (CKLFSF8)

Coagulation factor $\mathrm{C}$ (limulus polyphemus) homology (Coch-5B2)

Cystein-rich protein (CROIP) gene, exon 5, 6

deK (putative oncogen)

DLEC1 to DRCTL4 gene region section $1 / 2$

Down syndrome critical region protein A (DCAA)

Enhancer of rudimentary (Drosophila) homolog (ERH)

Finkel-Biskis-Reilly murin sarfoma virus (FBR-MuSV) ubiquitously expressed (fox derived)

Gene trap ankyrin repeat (GTAR)

HIV-1 TAR binding protein

HLA class III region containing NOTCH4 gene, homeobox

Jun D proto-oncogene (JCND)

KIAA0941 protein (= rab11-family interacting protein 2)

Kiss-1 metastasis-suppressor

Mu-crystallin gene, exon 8

NY57-antigen

Org/ALI-interacting protein Arg BO2 \& translated products (transcript varinat 2)

PAR (Parader-Willi/Angleman region) 5 gene from brain, 15q11-q13

Prohibin

Rearranged mRNA for glutamine synthase

Ring finger protein 11

S-adenosyl homocysteine hydrolase-like

Selenium binding protein 1

HUMIFP

NM_019059

NM003603

NM021069

XM_087254

HSA011117

XM_087402

$\mathrm{COCH}$

HUMCRP04

HS298J15

AB026898

NM006052

NM 004450

NM006522

AB014597

HUMTAP2

HSMHC3W5A

JUND

AB023158

NM002256

HSMVCRY6

AF1155114

NM021069

AF019618

PHB

X59834

AB024703

AHCYC1

NM_003944

$+$


Table 2. Continued

Gene description

Identical to
cochlear

Selenoprotein P, plasma 1 (SEPP1)

Serine (or cysteine) proteinase inhibitor, member 2

SET translation (myeloid leukemia-associated)

Similar to selenium binding protein 1

STX16 (syntaxin16)

Testis-specific Y-encoded-like protein (TSPYL)

The HMG2L 1 gene for high moleculity group protein 2-like 1

The N-O oct5a POU domain proteins

Thyroglobulin gene, exon 32

Thyroid autoantigen $70 \mathrm{kD}$ (ku antigen) (G22P1)

Transposon-derived Buster1 transposase-like protein

V-ski avian viral oncogene homolog

Wbscr1 and replication factor $\mathrm{C}$ subunit 2

\section{Unknown}

CGI-18 protein

cDNA DKFZp434H0115

cDNA DKFZp434E2023

cDNA DKFZP566N1346 (= DKFZp762K0911)

cDNA DKFZp586P1622

cDNA DKFZp686K0273

cDNA DKFZp686P072

cDNA DKFZp761F0621

cDNA FLJ 12116 (hypothetical protein)

cDNA FLJ 13794

cDNA FLJ 21778

cDNA FLJ 37657

Chromosome 6 open reading frame 1

Chromosome 11 open reading frame 15

Clone IMAGE:4042587, mRNA

Clone IMAGE:4052238, mRNA

Clone IMAGE:4097766, mRNA

Clone IMAGE:5528612, mRNA

Clone IMAGE:5752707, mRNA

HepG 3' region cDNA

Hypothetical protein CAB 56184

Hypothetical protein DKFZp564K0822

Hypothetical protein FLJ21016

Hypothetical protein FLJ21047

Hypothetical protein MGC 5350

KIAA0100 gene product

KIAA0537 gene product

KIAA0551 protein

KIAA0828 protein

Nop $10 \mathrm{p}$

Novel protein similar to predicted chicken protein

Similar to RIKEN cDNA 1110017 I16 gene

\begin{tabular}{lll} 
Occurrence & $\begin{array}{l}\text { Accession } \\
\text { no. }\end{array}$ & $\begin{array}{l}\text { Identical to } \\
\text { cochlear } \\
\text { ESTs }\end{array}$ \\
\hline 3 & SEPP1 & + \\
1 & BC015663 & \\
1 & NM003011 & \\
1 & BC009084 & \\
1 & HSJ907D15 & \\
1 & AF042181 & \\
1 & HS510H16 & \\
1 & HS273N12 \\
1 & HSTHRG08 \\
1 & XM010020 \\
1 & AF205600 \\
1 & SKI & \\
1 & AF045555 & \\
& &
\end{tabular}

AF151814
HSM801728
AL512761
AL110256
HSM800861
AL833622
AL832213
AL713742
AK022178
AK023856
AK025431
AK094976
AY062936
AJ400877
BC021694
BC014384
BC032596
BC016735
BC036789
D16932
BC014592
NM_030796
XM_015303
BC019890
XM_086221
XM_031460
AB011109
AB011123
XM_045904
AB043103
AL078581
BC018068

aspects in the fetal cochlea library database (http:// hearing.bwh.harvard.edu/cochlearcdnalibrary.htm\#EST production).

\section{Semiquantitative RT-PCR and inner-ear expression}

We performed RT-PCR experiments for the 22 selected EST clones of unknown function to confirm expression in the vestibule and eight other tissues. Nine of 22 ESTs were confirmed to be expressed in the vestibular tissue, as shown in Table 3. The expression of the two representative ESTs (clones 297 and 402) was confirmed in the inner ear (vestibule) and other organs (Fig. 1).

\section{Discussion}

We have reported here the successful construction of a human vestibular cDNA library by means of T7-based amplification of RNA. Although several methods were used previously for constructing mammalian inner-ear cDNA libraries, each library required a significant quantity of mRNA (Heller et al. 1998; Harter et al. 1999); for example, the well-established human fetal cochlear library required tissue from more than 100 fetal ears (Robertson et al. 1994; Skvorak et al. 1997). Because the collection of adequate amounts of inner ear tissue is so difficult, the number of clones obtained from a library will necessarily be limited. 
Table 3. Expression profile analysis of nine novel ESTs by RT-PCR

\begin{tabular}{|c|c|c|c|c|c|c|c|c|c|c|}
\hline Clone no. & Accession no. & Vestibule & Brain & Heart & Lung & Liver & Placenta & Kidney & Skeltetal muscle & Pancreas \\
\hline 18 & AB072818 & + & - & + & + & + & - & + & + & - \\
\hline $35^{\mathrm{a}}$ & AB072817 & + & + & - & - & + & + & - & - & - \\
\hline $36^{\mathrm{a}}$ & AB077323 & + & + & - & - & + & + & - & - & - \\
\hline 124 & AB072821 & + & + & + & + & + & + & + & + & - \\
\hline 129 & AB072822 & + & + & - & + & + & + & - & - & + \\
\hline 229 & AB072823 & + & + & + & - & + & + & + & + & + \\
\hline 297 & AB072825 & + & - & - & - & - & - & - & - & - \\
\hline 360 & AB074133 & + & + & + & + & - & + & + & - & - \\
\hline 402 & AB076958 & + & + & - & - & - & + & - & - & + \\
\hline
\end{tabular}

ESTs, Expressed sequence tags; RT-PCR, reverse transcriptase-polymerase chain reaction; + detected; - not detected

${ }^{a}$ No. 35 and 36 ESTs share the same nucleotid sequences, and are considered to be derived from the same gene

Fig. 1. Expression of vestibular cDNA clones (No. 297 and 402), estimated by reverse transcriptase-polymerase chain reaction (RT-PCR). A $2.5 \%$ agarose gel stained with ethidium bromide shows RT-PCR products obtained from aRNAs extracted from various tissues. One cycle of T7-based RNA amplification was performed in total RNA from the vestibule and eight other organs. Human glceraldehyde-3-phosphate dehydrogenase $(G A P D H)$ serves as a positive control for the RNA

No. 402 clone

\section{GAPDH}
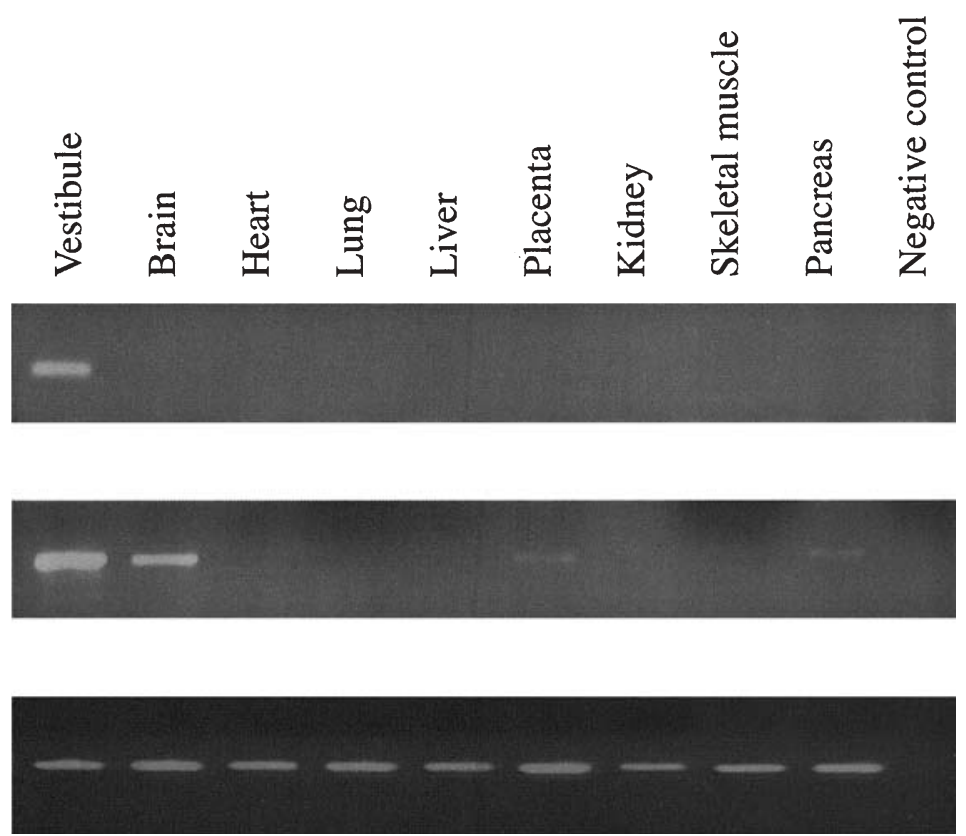

To overcome this primary disadvantage, we applied the T7based RNA-amplification method (Van Gelder et al. 1993; Luo et al. 1999). Our results demonstrated that information about gene expression in the vestibule could be obtained from an extremely small amount of RNA. Although insert sizes of selected clones from our vestibular cDNA library were not as large as those of cochlear cDNA libraries reported previously, the almost complete nature of our genomic sequence information made it easy to define the transcription unit. With regard to repetitive elements, the insufficient sequence complexity, similar to that described in Morton's cochlear cDNA library, might have been enhanced to produce excess artifacts, and all repetitive elements were excluded in this study. The T7-amplification method was able to amplify the transcripts by maintaining the proportion of the original mRNA species, and the T7amplified cDNA would be an almost complete representation of mRNA in vivo. Thus, identification of genes specifically expressed in the inner ear may be an effective approach to clarify which gene products play important roles in the auditory and vestibular systems and also to discover and characterize genes that carry mutations responsible for deafness. Characterization of our vestibular cDNA library showed a wide variety of molecules including many unknown function proteins, suggesting that yet additional gene products may be involved in the vestibular function. RT-PCR analyses of the selected 22 EST sequences showed that 9 ESTs were expressed in the inner ear. However, no expression of the other 13 ESTs was detected in any tissues, and the possibility cannot be excluded that this problem may be due to an artifact such as unexpected genomic sequence. To evaluate whether these 9 EST transcripts would be truly novel gene fragments, we need further investigation.

In the human, a few cochlear cDNA libraries have been reported (Robertson et al. 1999; Jacob et al. 1997), but until now no vestibular cDNA library was available. When we compared results from our vestibular cDNA library with those of the fetal cochlear library, we noted that many genes of known functions were common in both libraries. How- 
ever, dozens of genes detected in our vestibular library were not found in the fetal cochlear library, indicating that a specific set of genes is expressed in the vestibule.

Developmentally, the cochlea and vestibule originate from one embryonic organ, the otic capsule. The vestibular organ is remarkably similar to the cochlea, although their functions are very different. Some structures, including sensory hair cells, neuroepithelia, tectorial or otolithic membranes, and supporting cells, are common in both organs, suggesting that genes expressed in these two parts of the inner ear would play similar roles. Therefore, it is not surprising that the cDNA profile of our vestibular library significantly overlapped with that of the cochlear library. In fact, we encountered the $\mathrm{COCH}$ gene four times, the mutations of which are causative to nonsyndromic deafness with vestibular symptoms (DFNA9); this gene is reported to be highly expressed in the human cochlea and vestibule (Robertson et al. 1998). It is also of interest to know how vestibular function remains intact for many patients whose deafness is caused by mutations of nonsyndromic deafness genes expressed in both the vestibular and cochlear organs. Compared with the cochlear ESTs, particularly with respect to the phenotypic differences, the lack of vestibular dysfunction among affected patients who are carrying mutations in the deafness genes may reflect neurobiological differences based on gene expression profiling. The present study revealed only a small part of the human vestibular cDNA sequences. Nevertheless, the vestibular cDNA library that we have reported here represents a valuable experimental tool for investigating genes related to deafness and should contribute important insights into the molecular basis for the vestibular function.

Acknowledgments We thank all of the families whose participation made this project possible. We also thank Dr. Yasuhiro Inoue of Keio University School of Medicine for providing the surgical specimens. This work was supported in part by Research for the Future Program Grant \#00L01402 from the Japan Society for the Promotion of Science.

\section{References}

Fung K, Xie Y, Hall SF, Lillicrap DP, Taylor SA (2002) Genetic basis of familial Meniere's disease. J Otolaryngol 31:1-4
Harter C, Ripoll C, Lenoir R, Hamel CP, Rebillard G (1999) Expression pattern of mammalian cochlea outer hair cell (OHC): screening of a rat OHC cDNA library. DNA Cell Biol 18:1-10

Heller S, Sheane CA, Javed Z, Hudspeth AJ (1998) Molecular markers for cell types of the inner ear and candidate genes for hearing disorders. Proc Natl Acad Sci USA 95:11400-111405

Jacob AN, Baskaran N, Kandpal G, Narayan D, Bhargava AK, Kandpal RP (1997) Isolation of human ear specific cDNAs and construction of cDNA libraries from surgically removed small amounts of inner ear tissues. Somat Cell Mol Genet 23:83-95

Kelsell DP, Dunlop J, Stevens HP, Lench NJ, Liang JN, Parry G, Mueller RF, Leigh IM (1997) Connexin 26 mutations in hereditary non-syndromic sensorineural deafness. Nature 387:80-83

Luo L, Salunga RC, Guo H, Bittner A, Joy KC, Galindo JE, Xiao H, Rogers KE, Wan JS, Jackson MR, Erlander MG (1999) Gene expression profiles of laser-captured adjacent neuronal subtypes. Nat Med 5:117-122

Robertson NG, Khetarpal U, Gutierrez-Espeleta GA, Bieber FR, Morton CC (1994) Isolation of novel and known genes from a human fetal cochlear cDNA library using subtractive hybridization and differential screening. Genomics 23:42-50

Robertson, NG, Skvorak AB, Yin Y, Weremowicz S, Johnson KR, Kovatch KA, Battey JF, Bieber FR, Morton CC (1997) Mapping and characterization of a novel cochlear gene in human and in mouse: a positional candidate gene for a deafness disorder, DFNA9. Genomics 46:345-354

Robertson NG, Heller S, Lin JS, Resendes BL, Weremowicz S, Denis CS, Bell AM, Hudspeth AJ, Morton CC (2000) A novel conserved cochlear gene, OTOR: identification, expression analysis, and chromosomal mapping. Genomics 66:242-248

Skvorak AB, Robertson NG, YinY, Weremowicz S, Her H, Bieber FR, Beisel KW, Lynch ED, Beier DR, Morton CC (1997) An ancient conserved gene expressed in the human inner ear: identification, expression analysis, and chromosomal mapping of human and mouse antiquitin (ATQ1). Genomics 46:191-199

Skvorak AB, Weng Z, Yee AJ, Robertson NG, Morton CC (1999) Human cochlear expressed sequence tags provide insight into cochlear gene expression and identify candidate genes for deafness. Hum Mol Genet 8:439-452

Van Gelder RN, von Zastrow ME, Yool A, Dement WC, Barchas JD, Eberwine JH (1990) Amplified RNA synthesized from limited quantities of heterogeneous cDNA Proc Natl Acad Sci USA 87:16631667

Verhoeven K, Van Laer L, Kirschhofer K, Legan PK, Hughes DC, Schatteman I, Verstreken M, Van Hauwe P, Coucke P, Chen A, Smith RJ, Somers T, Offeciers FE, Van de Heyning P, Richardson GP, Wachtler F, Kimberling WJ, Willems PJ, Govaerts PJ, Van Camp G (1999) Mutations in the human alpha-tectorin gene cause autosomal dominant non-syndromic hearing impairment. Nat Genet 19:60-62

Yasunaga S, Grati M, Cohen-Salmon M, El-Amraoui A, Mustapha M, Salem N, El-Zir E, Loiselet J, Petit C (1999) A mutation in OTOF, encoding otoferlin, a FER-1-like protein, causes DFNB9, a nonsyndromic form of deafness. Nat Genet 4:363-369 